\title{
Model of Aeronautical Ground Lighting System Transformers
}

\author{
D. Vidal' ${ }^{1}$, LI. Monjo ${ }^{2}$, L. Sainz ${ }^{2}$ and J. Pedra ${ }^{2}$
}

1 A.A.S.A. Airport of Reus, Reus, 43206, Spain.

2 Department of Electrical Engineering, ETSEIB-UPC, Av. Diagonal 647, Barcelona 08028, Spain.

E-mails: dvlombarte@aena.es, Iluis.monjo@upc.edu, sainz@ee.upc.edu, pedra@ee.upc.edu

\begin{abstract}
Aeronautical ground lighting (AGL) systems provide visual reference to aircraft during airport operations. In AGL systems, constant current regulators feed a series circuit composed of luminaires supplied through transformers. Component modeling is necessary to simulate AGL systems, and thus characterize and predict their behavior. This paper presents an AGL transformer model including transformer core saturation. Moreover, a procedure to estimate transformer model parameters is proposed. Both the model and the estimation method are validated with extensive measurements on more than 20 AGL transformers of different power ratings and trade names.
\end{abstract}

\section{Nomenclature}

\begin{tabular}{|c|c|c|c|}
\hline$f$ & Frequency of the supply voltage & $T$ & Period of the supply voltage \\
\hline$\omega$ & Angular pulsation of the supply voltage & $R$ & Winding resistance of the transformer \\
\hline$R_{p}, R_{s}$ & Primary and secondary winding resistances & $X$ & Leakage reactance of the transformer \\
\hline$L_{d}$ & Leakage inductance of the transformer & $L_{d p}, L_{d s}$ & $\begin{array}{l}\text { Primary and secondary leakage } \\
\text { inductances }\end{array}$ \\
\hline$R_{F e}$ & Core loss resistance & $M$ & Core non-linear magnetizing inductance \\
\hline$u_{p}, u_{s}$ & Primary and secondary transformer voltages & $i_{p}, i_{s}$ & $\begin{array}{l}\text { Primary and secondary transformer } \\
\text { currents }\end{array}$ \\
\hline$i_{m}$ & Magnetizing current & $i_{F e}$ & Core loss resistance current \\
\hline$\phi$ & Core magnetic flux & $\lambda_{p}$ & $\begin{array}{l}\text { Core magnetic flux across the primary } \\
\text { winding }\end{array}$ \\
\hline$f_{t c}$ & Magnetic potential in the transformer core & $\Re\left(f_{t c}\right)$ & Non-linear reluctance \\
\hline
\end{tabular}




\begin{tabular}{|c|c|c|c|}
\hline$N_{p}, N_{s}$ & Primary and secondary winding turns & $r_{t}$ & Winding turn ratio \\
\hline$u_{p, \mathrm{sc}}$ & $\begin{array}{l}\text { Primary voltage measurements of the short- } \\
\text { circuit test }\end{array}$ & $i_{p, \mathrm{sc}}$ & $\begin{array}{l}\text { Primary current measurements of the } \\
\text { short-circuit test }\end{array}$ \\
\hline$u_{p, 0}, i_{p, 0}$ & $\begin{array}{l}\text { Primary voltage and current measurements } \\
\text { of the open-circuit test }\end{array}$ & $u_{s, 0}$ & $\begin{array}{l}\text { Secondary voltage measurements of the } \\
\text { open-circuit test }\end{array}$ \\
\hline$p_{F e}$ & Transformer active power consumption & $P_{N}$ & Rated power of the AGL transformer \\
\hline$D$ & Length of underground cables & $P_{B}$ & Active power of luminaires \\
\hline$R_{B}$ & \multicolumn{3}{|l|}{ Resistance of luminaires } \\
\hline $\mathbf{y}$ & \multicolumn{3}{|c|}{ Vector of magnetizing inductance function parameters } \\
\hline $\mathbf{r}(\mathbf{y})$ & \multicolumn{3}{|l|}{ Residual vector } \\
\hline $\mathbf{y}_{\mathbf{l b}}, \mathbf{y}_{\mathbf{u b}}$ & \multicolumn{3}{|c|}{ Lower and upper bound vectors of magnetizing inductance function parameters } \\
\hline$M_{1}, M_{2}, p$ & \multicolumn{3}{|l|}{ Parameters of the magnetizing inductance function } \\
\hline
\end{tabular}

\section{Introduction}

Aeronautical ground lighting (AGL) systems provide visual cues by means of luminaires to aircraft pilots during approach, landing and taxiing [1]. These systems are $5 \mathrm{kV}$ series circuits of $1 \times 6 \mathrm{~mm}^{2}$ copper insulated underground cables where constant current regulators (CCRs) supply luminaires through transformers (Fig. 1a) [1]-[5]. Constant current regulators are variable voltage sources close to the airport power supply which provide an adjustable rms current according to brightness requirements. These regulators modify the supply voltage to maintain the desired rms current and ensure circuit continuity, even in case of luminaire burnt out. Their standard power ratings are $4,7.5,10,15,20,25,30,50$ and $70 \mathrm{kVA}$ with a typical current rating of $6.6 \mathrm{~A}$ (a current rating of $20 \mathrm{~A}$ is also common for AGL systems with power ratings above $30 \mathrm{~kW}$ ). Currents have five discrete brightness steps $(6.6,5.2,4.1,3.4$ and $2.8 \mathrm{~A}$ or 20.0, 15.8, 12.4, 10.3 and 8.5 A) [1], [2], [4]. Luminaires are visual aids for pilots. Their pattern, intensity, color and direction of light emission are modified according to use (approach lighting system (ALS), precision approach path indicator (PAPI), runway lighting system (RLS) and taxiway lights and guidance signs). They are generally classified into high and low intensity luminaires. The former are used for ALS, PAPI and RLS and the latter for taxiway lights and guidance signs [1], [2], [6]. The number of luminaires ranges from 10 up to 300 in small and large AGL systems, respectively $\left(N_{b}=10\right.$ to 300 in Fig. 1a). AGL transformers separate CCRs and luminaires into primary and secondary circuits. They ensure continuity of the series circuit even in case of luminaire burnt out (allowing functioning luminaires to remain lit) and isolate luminaires from the high operating voltage of the primary circuit (generally insulated to $5 \mathrm{kV}$ ). In the event of luminaire burnt out, the transformers maintain circuit continuity but work in saturation because they operate in open circuit at series circuit rated current. This must be considered in AGL system studies (i.e., in AGL transformer modeling) because saturation modifies the CCR operating conditions and could reduce AGL system power quality [7].

AGL systems require frequent expensive maintenance and conservation work mainly consisting in the detection and location of failed luminaires [5]. AGL simulation tools and component models are necessary to study the behavior of these systems and thus predict their response to electrical events or further technological 


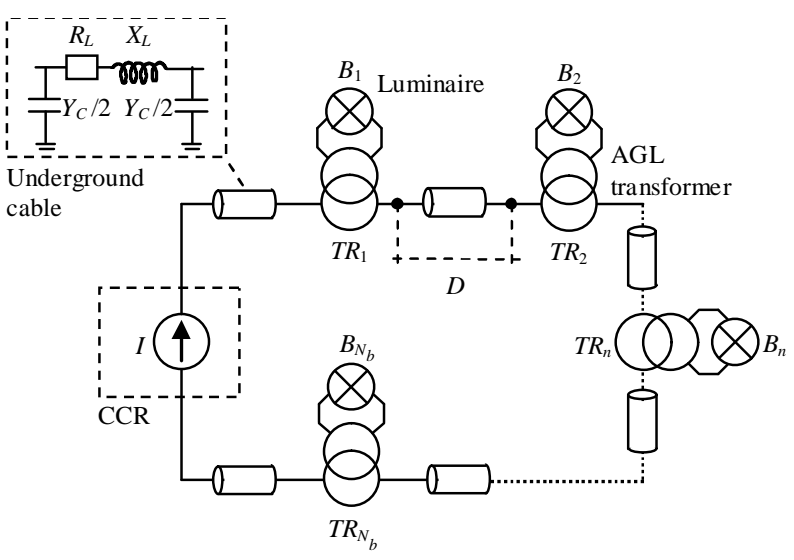

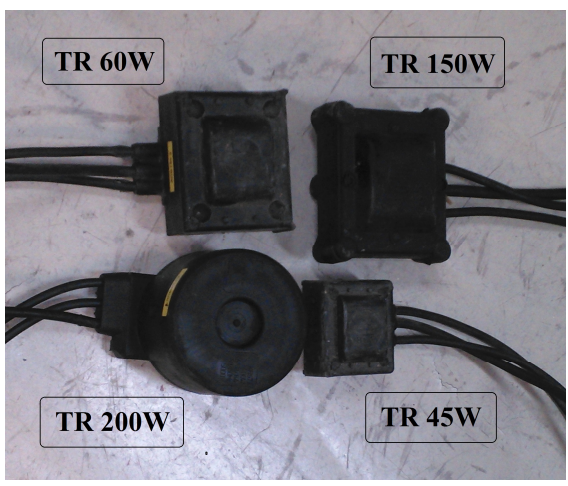

$b$

$a$

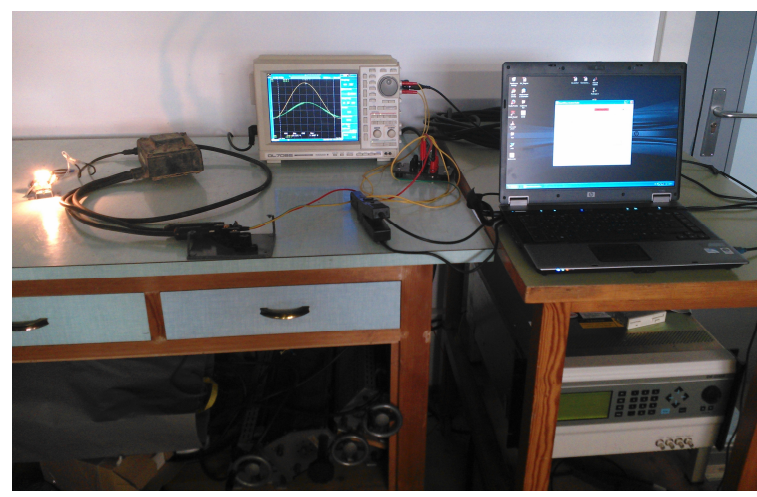

$c$

Fig. 1

a AGL system

b Isolation transformers

c Setup of circuit measurements

changes in the system components [4], [5], [8], [9]. Procedures for AGL component parameter estimation are also required. However, there is still a lack of adequate AGL system component models for performing accurate simulations of AGL system electrical response.

This paper provides results of measurements on more than 20 actual AGL transformers delivered by the airport of Reus (Spain) to the power quality laboratory of the Department of Electrical Engineering, Universitat Politècnica de Catalunya (UPC). A model considering the transformer saturation characteristics is presented from the traditional transformer equivalent circuit. The paper also proposes a method for model parameter estimation. Both are validated with extensive measurements.

\section{AGL transformer model}

AGL transformers are single-phase transformers made as fully encapsulated units with their own primary and secondary cables (Fig. 1b). They generally consist of a rectangular magnetic core made of low-loss grain oriented laminations and with separated primary and secondary windings made of enameled copper wire (Fig. 2a). Their power ratings range from 30 to $500 \mathrm{~W}$ and their current ratio is generally 6.6/6.6 A (i.e., the winding turn ratio $r_{t}$ is equal to 1). Other turn ratios (e.g., 20/6.6 A) are common in AGL systems with power ratings above $30 \mathrm{~kW}$ because the CCR current is greater than $6.6 \mathrm{~A}$ to maintain the primary circuit voltage below $5 \mathrm{kV}$. 


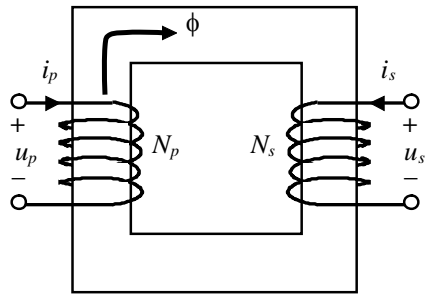

$\boldsymbol{a}$
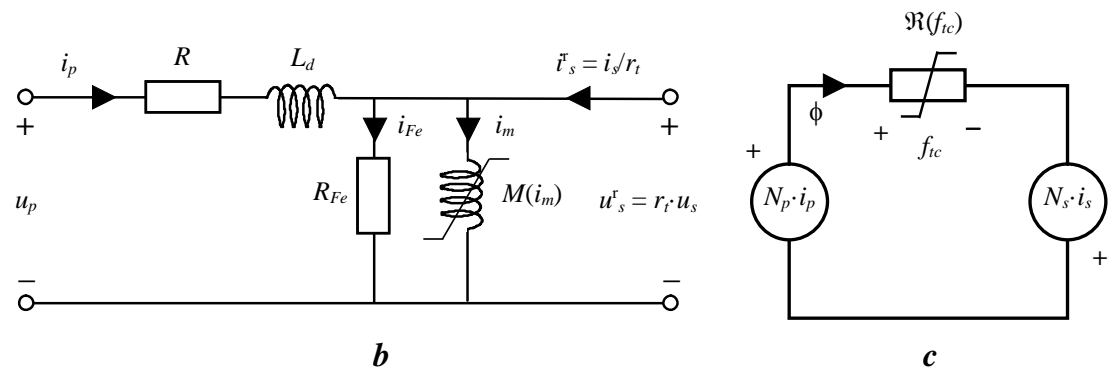

$c$

Fig. 2 Equivalent circuits of AGL transformers:

a AGL transformer

b Electric equivalent circuit

c Magnetic equivalent circuit.

\subsection{Electric circuit modeling}

Fig. $2 \mathrm{~b}$ shows the electric equivalent circuit of AGL transformers, where $R=R_{p}+r_{t}^{2} \cdot R_{s}$ and $L_{d}=L_{d p}+r_{t}^{2} \cdot L_{d s}$ are the primary/secondary winding resistance and constant leakage inductance, $R_{F e}$ is the core loss resistance and $M\left(i_{m}\right)$ is the core non-linear magnetizing inductance, which depends on the magnetizing current $i_{m}$. According to Fig. 2b, the electric relations of transformers are

$$
\begin{aligned}
& u_{p}=\left(R+L_{d} \frac{d}{d t}\right) i_{p}+r_{t} \cdot u_{s} \quad u_{s}=\frac{1}{r_{t}} \frac{d \lambda_{p}}{d t}=\frac{1}{r_{t}} \frac{d\left(M\left(i_{m}\right) \cdot i_{m}\right)}{d t} \\
& 0=R_{F e} \cdot i_{F e}-\frac{d\left(M\left(i_{m}\right) \cdot i_{m}\right)}{d t} \\
& i_{m}=i_{p}+i_{s} / r_{t}-i_{F e},
\end{aligned}
$$

where $r_{t}=N_{p} / N_{s}$ is the winding turn ratio, $N_{p}$ and $N_{s}$ are the primary and secondary winding turns and $\lambda_{p}=M\left(i_{m}\right) \cdot i_{m}$ is the core magnetic flux across the primary winding.

\subsection{Magnetic Circuit Modeling}

Fig. 2c shows the magnetic circuit of AGL transformers, which provides the magnetic flux and current relations:

$$
\begin{aligned}
& N_{p} \cdot i_{p}+N_{s} \cdot i_{s}=f_{t c} \\
& f_{t c}=\mathfrak{R}\left(f_{t c}\right) \cdot \phi,
\end{aligned}
$$

where $N_{p} \cdot i_{p}, N_{s} \cdot i_{s}$ are the primary/secondary magnetomotive forces, $f_{t c}$ is the magnetic potential in the transformer core, $\phi$ is the core magnetic flux and $\mathfrak{R}\left(f_{t c}\right)$ is the non-linear reluctance, which depends on the magnetic potential in the core. By neglecting the current flowing through the core loss resistance compared to the primary and secondary currents (i.e., $\left.i_{m} \approx i_{p}+i_{s} / r_{t}\right)$, (2) can be arranged as follows to obtain the $\left(\lambda_{p}-i_{m}\right)$ core saturation curve:

$$
i_{p}+\frac{i_{s}}{r_{t}}=\frac{\Re\left(f_{t c}\right)}{N_{p}} \cdot \frac{\lambda_{p}}{N_{p}} \Rightarrow \lambda_{p} \approx N_{p}^{2} \cdot \Re^{-1}\left(f_{t c}\right) \cdot i_{m}=M\left(i_{m}\right) \cdot i_{m}
$$




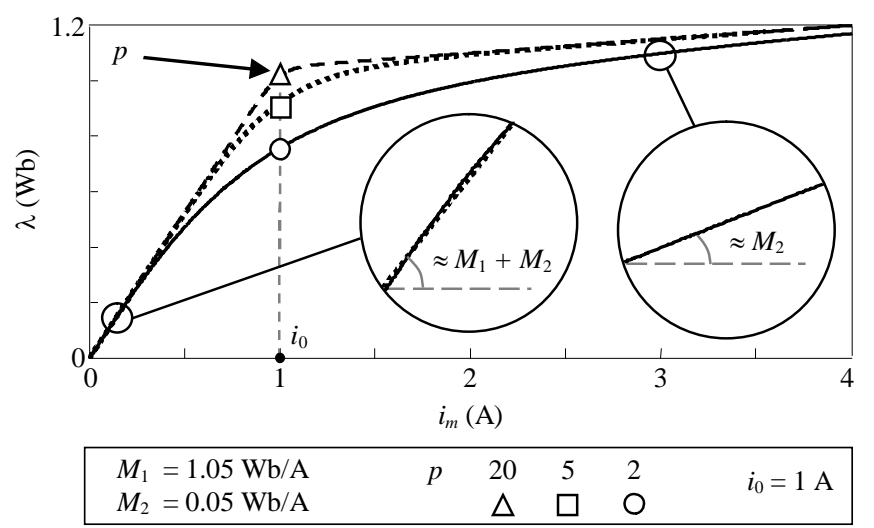

Fig. $3\left(\lambda_{p}-i_{m}\right)$ characteristic of the proposed core saturation curve.

The following functional relationship is used to characterize the $\left(\lambda_{p}-i_{m}\right)$ core saturation curve $M\left(i_{m}\right)$ by means of an anhysteretic magnetization curve:

$$
M\left(i_{m}\right)=\frac{M_{1}}{\left(1+\left(i_{m} / i_{0}\right)^{p}\right)^{1 / p}}+M_{2}
$$

where $M_{1}, M_{2}, p$ and $i_{0}$ are experimental parameters allowing the fitting of the above reluctance function to the $\left(\lambda_{p}-i_{m}\right)$ core saturation curve (see Fig. 3$)$ :

- $M_{1}$ and $M_{2}$ define the slope of the linear and non-linear segments of the core saturation curve.

- $p$ defines the shape of the curve.

- $i_{0}$ defines the core saturation knee point.

\section{AGL transformer parameter estimation}

Studies on AGL systems with the transformer model in the previous Section estimate the equivalent circuit parameters $R, L_{d}, R_{F e}$ and the core magnetizing inductance $M\left(i_{m}\right)$ (4) from voltage and current measurements.

\subsection{Transformer Winding Parameters}

The voltage and current measurements of the short-circuit test (i.e., $u_{p}$, sc and $i_{p \text {, sc }}$ ) provide a fairly accurate estimate of the transformer winding resistance and leakage inductance. In this test, the secondary voltage is zero (i.e., $\left.u_{s, \mathrm{sc}}=0\right)$ and (1) results in

$$
u_{p, s c}=\left(R+L_{d} \frac{d}{d t}\right) i_{p, s c} \Rightarrow \underline{U}_{p, s c}=\left(R+j L_{d} \cdot \omega\right) \cdot \underline{I}_{p, s c}
$$

where $\omega=2 \pi \cdot f$ and $f$ is the frequency of the sinusoidal supply voltage of the short-circuit test, $u_{p, s c}$.

\subsection{Transformer Core Parameters}

The voltage and current measurements of the open-circuit test (i.e., $u_{p, 0}, u_{s, 0}$ and $i_{p, 0}$ ) provide an estimate of 
the transformer core resistance and anhysteretic magnetization curve (4).

The core resistance is estimated from the transformer active power balance in the open-circuit test

$$
p_{F e}=\frac{\left(r_{t} \cdot u_{s, 0}\right)^{2}}{R_{F e}}=u_{p, 0} \cdot i_{p, 0}-R_{p} \cdot i_{p, 0}^{2} \Rightarrow R_{F e}=\frac{\left(r_{t} \cdot U_{s, 0}\right)^{2}}{\left\{u_{p, 0} \cdot i_{p, 0}\right\}_{\text {mean }}-R_{p} \cdot I_{p, 0}^{2}}, \text { (6) }
$$

where $I_{p, 0}$ and $U_{s, 0}$ are the rms values of the primary current and the secondary voltage of the open-circuit test, respectively. In the previous expression, the primary winding resistance $R_{p}$ is approximated to half the shortcircuit resistance value (i.e., $R_{p} \approx R / 2$ ).

Parameters $M_{1}, M_{2}, p$ and $i_{0}$ of the single-valued function $M\left(i_{m}\right)$ in (4) are estimated by close fitting of the open-circuit measurements of the $\left(\lambda_{p}-i_{m}\right)$ core saturation curve. Considering that the secondary current is zero, the measured magnetizing current and flux are evaluated as follows

$$
\begin{aligned}
& i_{m, 0}=i_{p, 0}-\frac{r_{t} \cdot u_{s, 0}}{R_{F e}} \\
& u_{s, 0}=\frac{1}{r_{t}} \frac{d \lambda_{p, 0}}{d t} \Rightarrow \lambda_{p, 0}(t)=r_{t} \cdot \int_{0}^{t} u_{s, 0}(t) \cdot d \tau+\lambda_{p r}
\end{aligned}
$$

where $\lambda_{p r}$ is the residual flux, which is calculated by imposing that

$$
\int_{0}^{T} \lambda_{p, 0}(t) \cdot d \tau=0 \quad(T=1 / f)
$$

with $f$ being the frequency of the sinusoidal supply voltage open-circuit test, $u_{p, 0}$. Subsequently, the singlevalued $\left(\lambda_{p}-i_{m}\right)$ function in (4) is determined from the measured $\left(\lambda_{p}-i_{m}\right)$ curve as

$$
M_{\text {meas }}\left(i_{m, 0}\right)=\frac{\lambda_{p, 0}}{i_{m, 0}}
$$

and parameters $M_{1}, M_{2}, p$ and $i_{0}$ in (4) are estimated by solving a non-linear least-square algorithm formulated as follows:

$$
\begin{aligned}
& \underset{\mathbf{y}}{\min }(S(\mathbf{y}))=\min _{\mathbf{y}}\left(r(\mathbf{y})^{T} r(\mathbf{y})\right) \\
& \text { subject to } \\
& \mathbf{y}_{\mathbf{l b}} \leq \mathbf{y} \leq \mathbf{y}_{\mathbf{u b}},
\end{aligned}
$$

where $\mathbf{y}=\left(M_{1}, M_{2}, p\right.$ and $\left.i_{0}\right), \mathbf{y}_{\mathbf{l b}}$ and $\mathbf{y}_{\mathbf{u b}}$ are vectors representing the lower and upper bounds of the parameters and $r(\mathbf{y})$ is the residual vector corresponding to differences between samples of the measured and simulated transformer magnetization curves (i.e., the measured and simulated functions $M_{\text {meas }}\left(i_{m}\right)$ and $M\left(i_{m}\right)$ in (9) and (4), respectively) at each measured current value $i_{m, 0}$, 


\begin{tabular}{|c|c|c|c|c|c|c|c|c|c|}
\hline & \multirow{2}{*}{\multicolumn{2}{|c|}{ Technical data }} & \multirow[b]{3}{*}{ Supplied load } & \multicolumn{6}{|c|}{ Estimated parameters } \\
\hline & & & & \multicolumn{2}{|c|}{$\begin{array}{c}\text { Winding } \\
\text { parameters }\end{array}$} & \multicolumn{4}{|c|}{ Core parameters } \\
\hline & Manufacturer & $\begin{array}{l}P_{N} \\
(\mathrm{~W})\end{array}$ & & $\begin{array}{c}R \\
(\Omega)\end{array}$ & $\begin{array}{c}L_{d} \\
(\mathrm{mH})\end{array}$ & $\begin{array}{c}M_{1} \\
(\mathrm{mH})\end{array}$ & $\begin{array}{c}M_{2} \\
(\mathrm{mH})\end{array}$ & $\begin{array}{c}p \\
(\mathrm{pu})\end{array}$ & $\begin{array}{l}i_{0} \\
\text { (A) }\end{array}$ \\
\hline TR $60 \mathrm{~W}$ & $\# 2$ & 60 & $\begin{array}{l}\text { Signal area and sign panels and } 45 \mathrm{~W} \text { low intensity luminaires far from } \\
\text { AGL transformers }\end{array}$ & 0.281 & 0.849 & 327.6 & 1.40 & 1.20 & 0.321 \\
\hline TR $150 \mathrm{~W}$ & $\# 1$ & 150 & $150 \mathrm{~W}$ high intensity luminaires for ALS, PAPI and RLS & 0.281 & 1.19 & 693.8 & 1.80 & 0.906 & 0.286 \\
\hline TR 200W & \# 3 & 200 & $200 \mathrm{~W}$ high intensity luminaires for ALS, PAPI and RLS & 0.301 & 1.71 & 650.0 & 1.80 & 1.15 & 0.338 \\
\hline
\end{tabular}

$$
r(\mathbf{y})=\left[\begin{array}{c}
\vdots \\
M_{\text {meas }}\left(i_{m, 0}\right)-M\left(i_{m, 0}\right) \\
\vdots
\end{array}\right] .
$$

The above non-linear least-square problem is solved by the MATLAB function lsqnonlin $(\cdot)$ [10] considering the following parameter bounds:

- $M_{1}=\left(0, M_{1, u b}\right)$, where the upper limit value $M_{1, u b}$ is set to $1 \mathrm{H}$ according to the experimental test results.

- $M_{2}=\left(0, M_{2, u b}\right)$, where the upper limit value $M_{2, u b}$ is set to $3 \mathrm{mH}$ according to the experimental test results.

- $p=(0,50)$, where upper limit values above 20 do not affect the solution of the least-square problem.

- $i_{0}=\left(0, i_{0, u b}\right)$, where the upper limit value $i_{0, u b}$ is chosen by simple inspection of the $\left(\lambda_{p}-i_{m}\right)$ core saturation curve.

The initial values of the non-linear least-square problem are the upper limit values of the parameters divided by 2 .

\section{$4 \quad$ Experimental Validation of the Model}

In order to validate experimentally the proposed transformer model, several laboratory tests were conducted on four 6.6/6.6 A 50 Hz AGL transformers of different power ratings and trade names (Fig. 1b and Table 1). These transformers, delivered by the airport of Reus (Spain), are ICAO and FAA compliant [2], [11]. Transformers of 300 and $400 \mathrm{~W}$ power ratings were also tested. However, the experimental measurements revealed that their behavior cannot be accurately described by the proposed model. Further studies (out of the scope of this paper) are being performed to understand their behavior and find an appropriate model for this type of transformers. The short- and open-circuit tests were first carried out to estimate the transformer parameters, followed by two tests to determine the accuracy of the model. The test circuits were fed with the power sources $15 \mathrm{kVA}$ AC Spitzerberger Three-phase Switching Amplifier or $4.5 \mathrm{kVA}$ AC ELGAR Smartwave Switching Amplifier, which can generate adjustable voltage waveforms. Recordings were made with a YOKOGAWA DL 708E digital scope. The test set-up consisted of the power supply feeding the test circuits and the oscilloscope suitably connected for measurements (Fig. 1c). Simulations to validate the model were performed with 
Matlab/Simulink software [10].

\subsection{AGL Transformer Parameter Estimation}

Five units of the four AGL transformers in Table 1 were tested to obtain their parameters. The mean of the estimate was adopted as the final parameter value of each transformer type. These mean values are summarized in Table 1.

The winding resistance and leakage inductance were estimated by the short-circuit test at transformer rated current. As an example, Fig. 4a plots the measured supply voltage and current waveforms of one unit of the $60 \mathrm{~W}$ transformer. Fig. 4b shows the estimated parameter values for the five units of each transformer type and the mean value of the estimated parameters. It can be observed that the winding parameters of the different units are rather similar and the mean value is a good estimate of these parameters. To validate the estimates, the simulated current waveform of the $60 \mathrm{~W}$ transformer is also shown with broken lines in Fig. 4b.The same accurate results were obtained for the other transformers.

The core resistance and parameters of the $\left(\lambda_{p}-i_{m}\right)$ core saturation curve (4) were estimated by the open-circuit

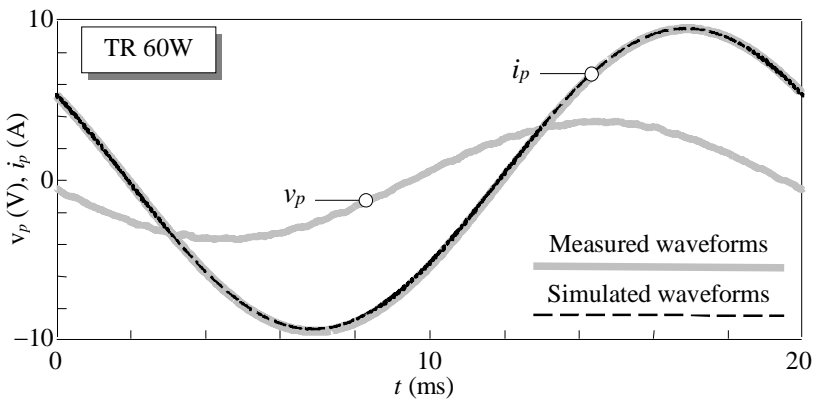

$a$

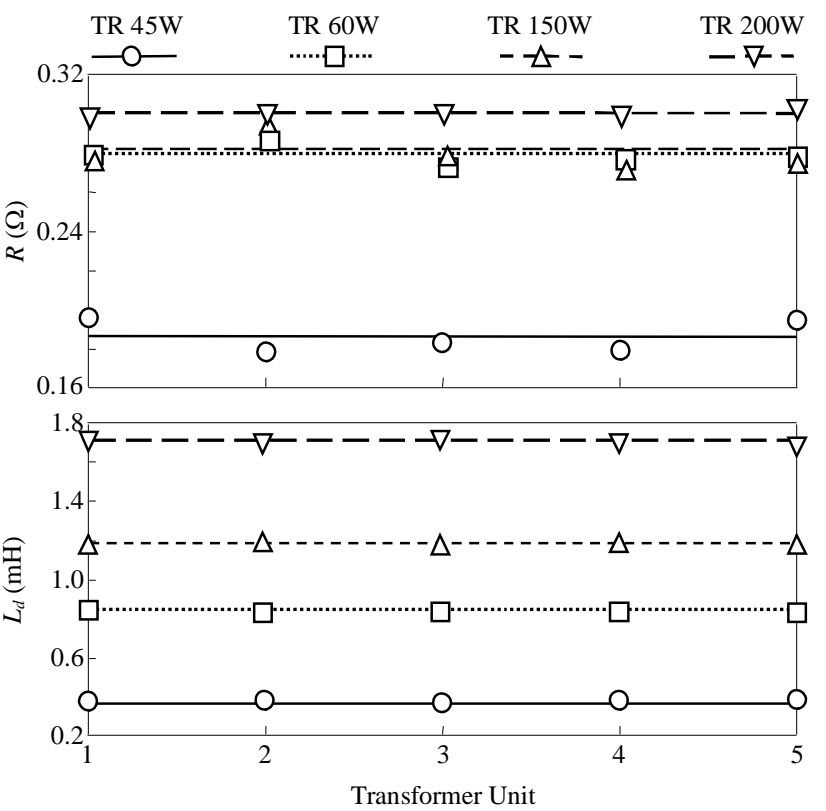

$\boldsymbol{b}$

Fig. 4

a Measured and simulated voltage and current waveforms in the short-circuit test.

$\boldsymbol{b}$ Transformer winding parameter estimation. 

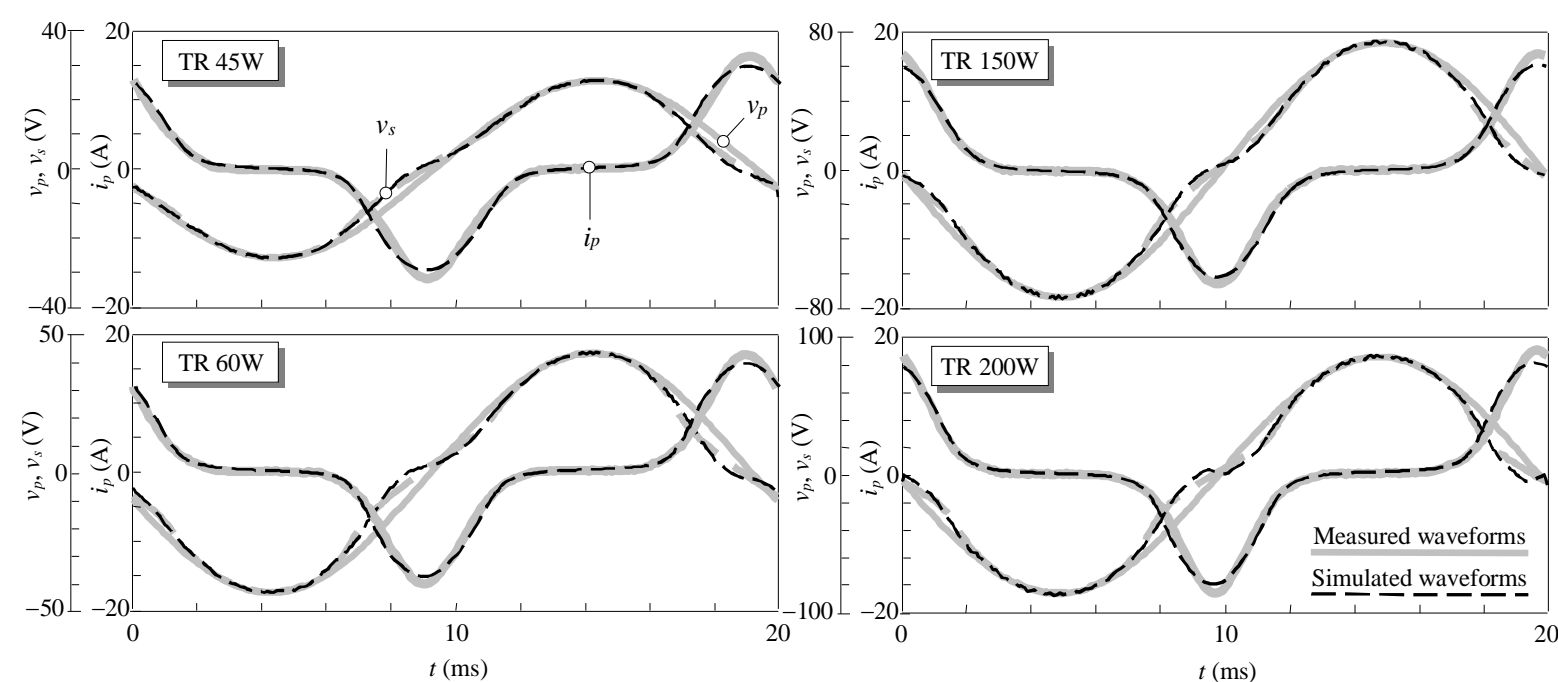

Fig. 5 Measured and simulated voltage and current waveforms in the open-circuit tests.
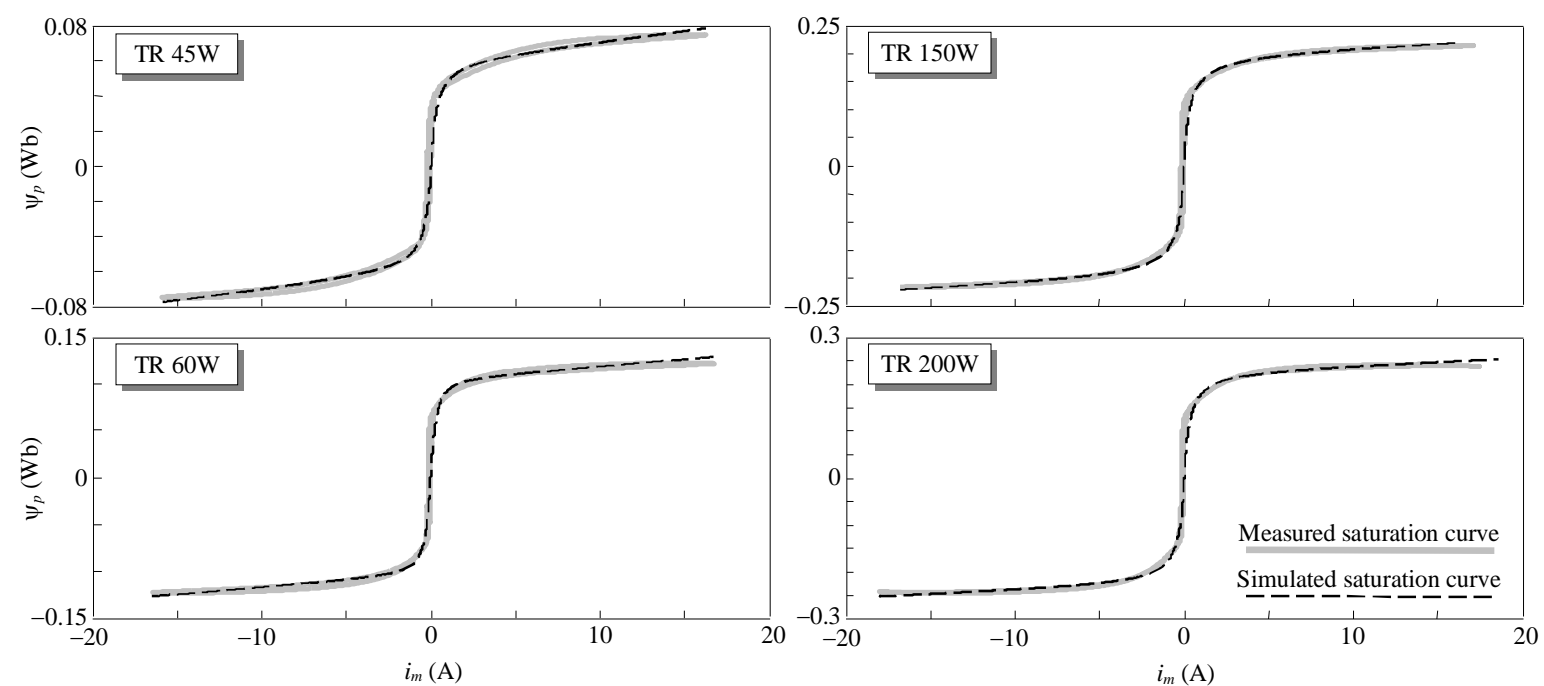

Fig. 6 Measured $\left(\lambda_{p}-i_{m}\right)$ core saturation curve obtained in the open-circuit tests and simulated $\left(\lambda_{p}-i_{m}\right)$ single-valued saturation curve.

test at 1.15 times the transformer rated current. As an example, Fig. 5 and Fig. 6 plot the measured voltages and current waveforms and the experimental $\left(\lambda_{p}-i_{m}\right)$ core saturation curves of one unit of each AGL transformer type, respectively. It can be noted that the area of the $\left(\lambda_{p}-i_{m}\right)$ hysteresis loops in Fig. 6 is very small, and therefore the core resistance $R_{F e}$ is very large and may be neglected in the electric circuit of Fig. 2b. Fig. 7 shows the estimated parameter values of the $\left(\lambda_{p}-i_{m}\right)$ core saturation curve for the five units of each transformer type and the mean value of the estimated parameters. Although the parameter values of the saturation curves vary more than short-circuit parameter values, they can also be represented by their mean value. To validate the estimate, the simulated voltage and current waveforms and $\left(\lambda_{p}-i_{m}\right)$ core saturation curves are also shown with broken lines in Fig. 5 and Fig. 6, respectively. As can be seen, they are acceptably accurate.

\subsection{AGL Transformer Model Validation}

Two tests were conducted to validate the AGL transformer model:

- Test \#1: The series circuit with five luminaires fed through AGL transformers in Fig. 8a was supplied with sinusoidal voltage and 6.6 A rms current. 


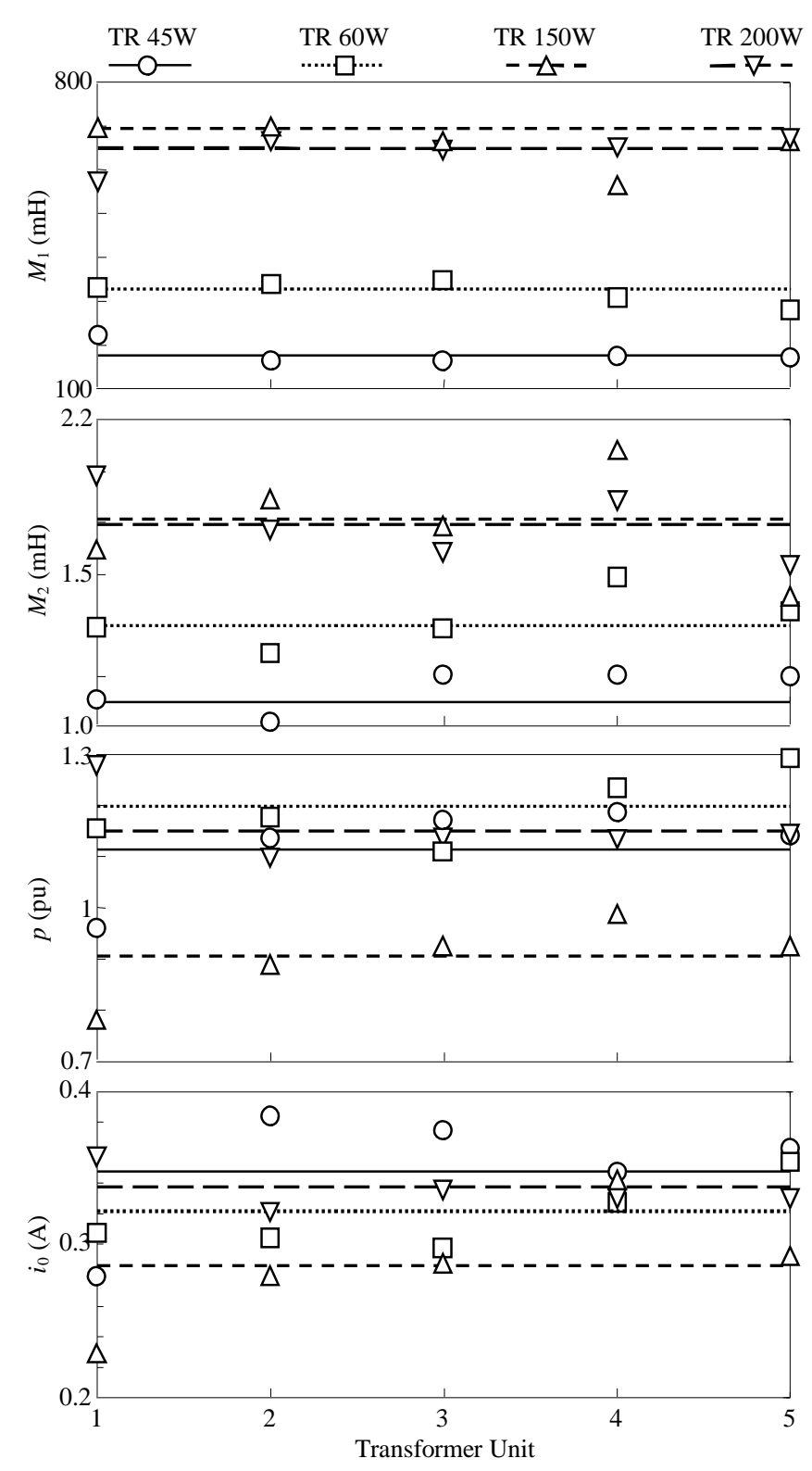

Fig. 7 Measured $\left(\lambda_{\mathrm{p}}-\mathrm{i}_{\mathrm{m}}\right)$ core saturation curve obtained in the open-circuit tests and simulated $\left(\lambda_{\mathrm{p}}-\mathrm{i}_{\mathrm{m}}\right)$ single-valued saturation curve.

- Test \#2: The circuit representing an AGL system with $N_{b}$ luminaires in Fig. 8b was supplied with sinusoidal voltage and $6.6 \mathrm{~A}$ rms current. Only one luminaire was connected in this circuit and the others (together with the underground cables and the AGL transformers) were modeled with the resistance $R_{\mathrm{Eq}}\left(N_{b}\right)$. Considering that the reactance-to-resistance ratio of the set formed by the cable, AGL transformer and luminaire is below $10 \%$, the resistance $R_{\mathrm{Eq}}\left(N_{b}\right)$ models approximately the impedance of $\left(N_{b}-1\right)$ sets, i.e.,

$$
R_{E q}=\left(N_{b}-1\right) \cdot \sqrt{\left(D \cdot R_{L}+R+P_{B} / 6.6^{2}\right)^{2}+\left(D \cdot X_{L}+X\right)^{2}},
$$

where $R_{L}, X_{L}$ and $D$ are the resistance, reactance and length of the underground cables (Fig. 1), $R$ and $X=L_{d^{*}} \omega$ are the winding resistance and leakage reactance of the AGL transformers (Table 1) and $P_{B}$ is the active power of the luminaires. Typical values were used for the cable parameters $\left(R_{L}=3.08 \Omega / \mathrm{km}, X_{L}=0.172 \Omega / \mathrm{km}\right.$ and 


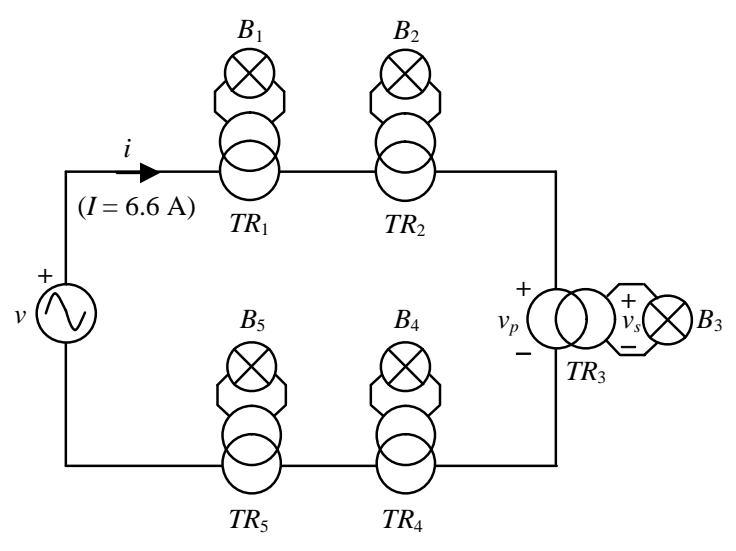

$\boldsymbol{a}$

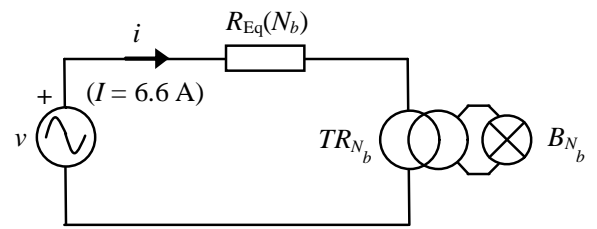

b

Fig. 8 Circuit tests:

a Test \#1: Series circuit with five luminaires fed through AGL transformers.

b Test \#2: Circuit representing an AGL system with $N_{b}$ luminaires.

$D=30 \mathrm{~m})$.

In both tests, the AGL transformers fed luminaires of the same power rating except for $60 \mathrm{~W}$ transformers, which fed $45 \mathrm{~W}$ luminaires because there are no $60 \mathrm{~W}$ luminaires (these transformers are used to feed other load types, Table 1). Voltage and current measurements were made and compared with simulations using the proposed transformer model and the estimated model parameters in Table 1. The AGL system luminaires were modeled as a resistance $R_{B}$ because they are usually halogen lamps. It was experimentally verified that their value can be accurately calculated from the luminaire active power and the current rating of the highest brightness step (i.e., $R_{B}=P_{B} / 6.6^{2}$ in (12)). Both tests were also performed for the other discrete brightness steps (i.e., 5.2, 4.1, 3.4 and 2.8 A) with similar accurate results (not shown for the sake of space).

\subsubsection{Results of test \#1}

The circuit of test \#1 was checked with all the luminaires on and with one, two and three failed luminaires. The circuit consumed current $i$ and the secondary voltage $v_{s}$ of one AGL transformer with one failed luminaire were measured. Fig. 9 summarizes the measurements and simulations of this current and voltage. The circuit supply voltage is also plotted as a reference. The fair accuracy of the proposed model is worth noting. The tests show that a large number of failed luminaires leads to a sharp consumed current waveform and increases harmonic current distortion. This is investigated in the next Subsection. Fig. 10 shows the simulations of the voltage distribution in the primary windings of the $150 \mathrm{~W}$ transformers. The circuit supply voltage is also plotted for comparison purposes. Although measurements are not shown for the sake of simplicity, the accuracy of the simulated results was also experimentally verified. The following observations can be made from Figs. 11 and 12: (i) Primary and secondary voltages are approximately equal because the winding turn ratio of AGL transformers is equal to 1 and the winding impedance is small. (ii) Voltage waveforms at terminals of the AGL transformers with non-failed luminaires are similar than consumed current waveforms because they are approximately equal to the product of the currents by the luminaire resistance. (iii) When only one luminaire fails, the AGL transformer of this luminaire can support high primary voltages because the main voltage drop in the series circuit occurs in its equivalent impedance (this problem is not as severe if more luminaires fail because the voltage drop is shared between their transformers). Similar results were obtained for the other transformers. 

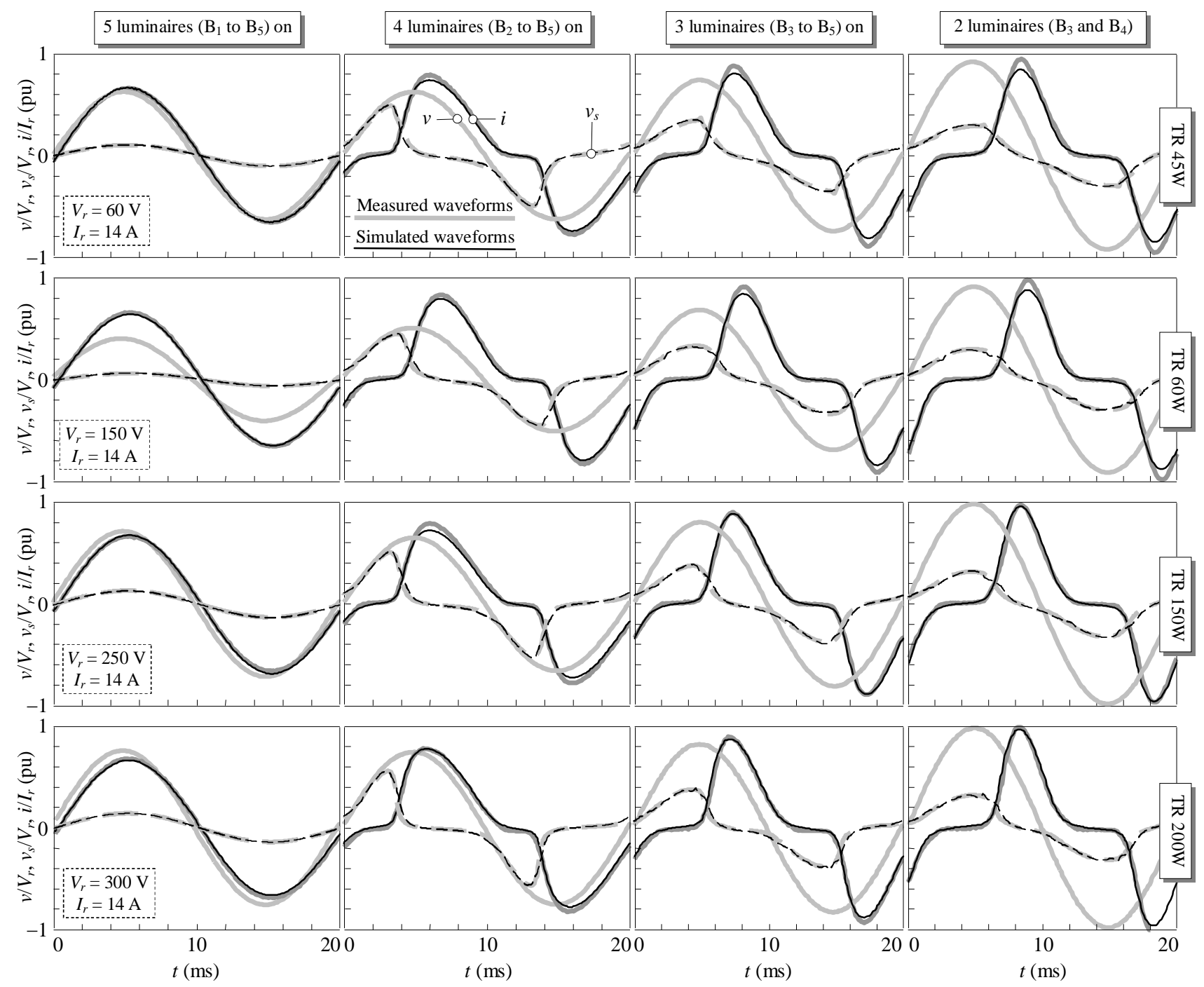

Fig. 9 Measured and simulated voltage and current waveforms of Test \# 1 .

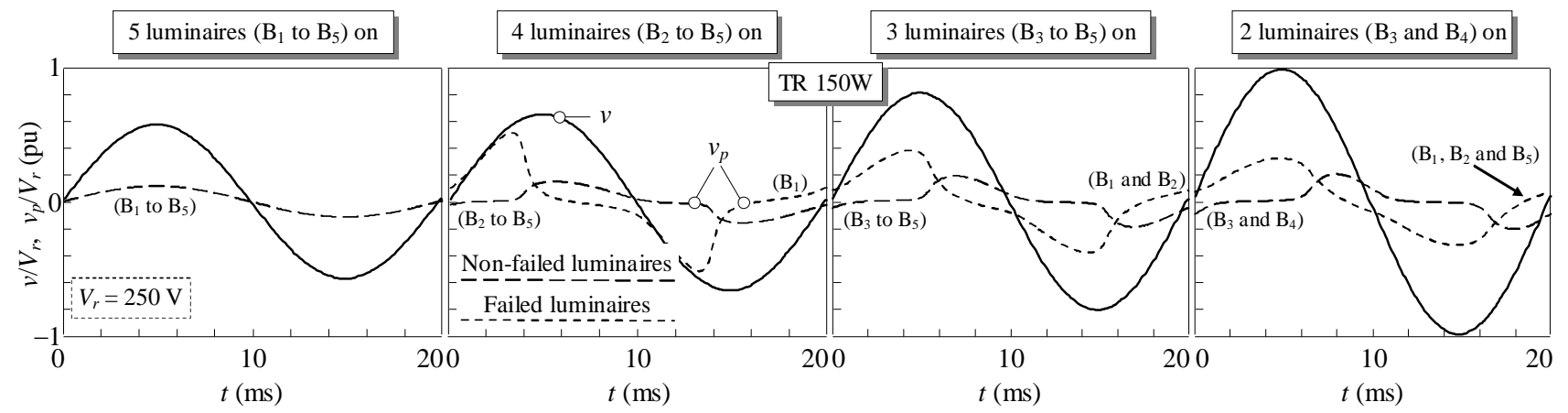

Fig. 10 AGL transformer primary voltages of Test \# 1.

Note that it is difficult to measure the impact of luminaire burnt out on the voltage distribution in all the primary windings of the AGL transformers. Simulations allow this concern to be easily analyzed.

\subsubsection{Results of test \#2}

The circuit of test \#2 was checked in the laboratory for different $R_{\mathrm{Eq}}\left(N_{b}\right)$ values (i.e., for AGL systems with different numbers of luminaires, $N_{b}$ ) and with one luminaire on or off. The circuit consumed current $i$ was 

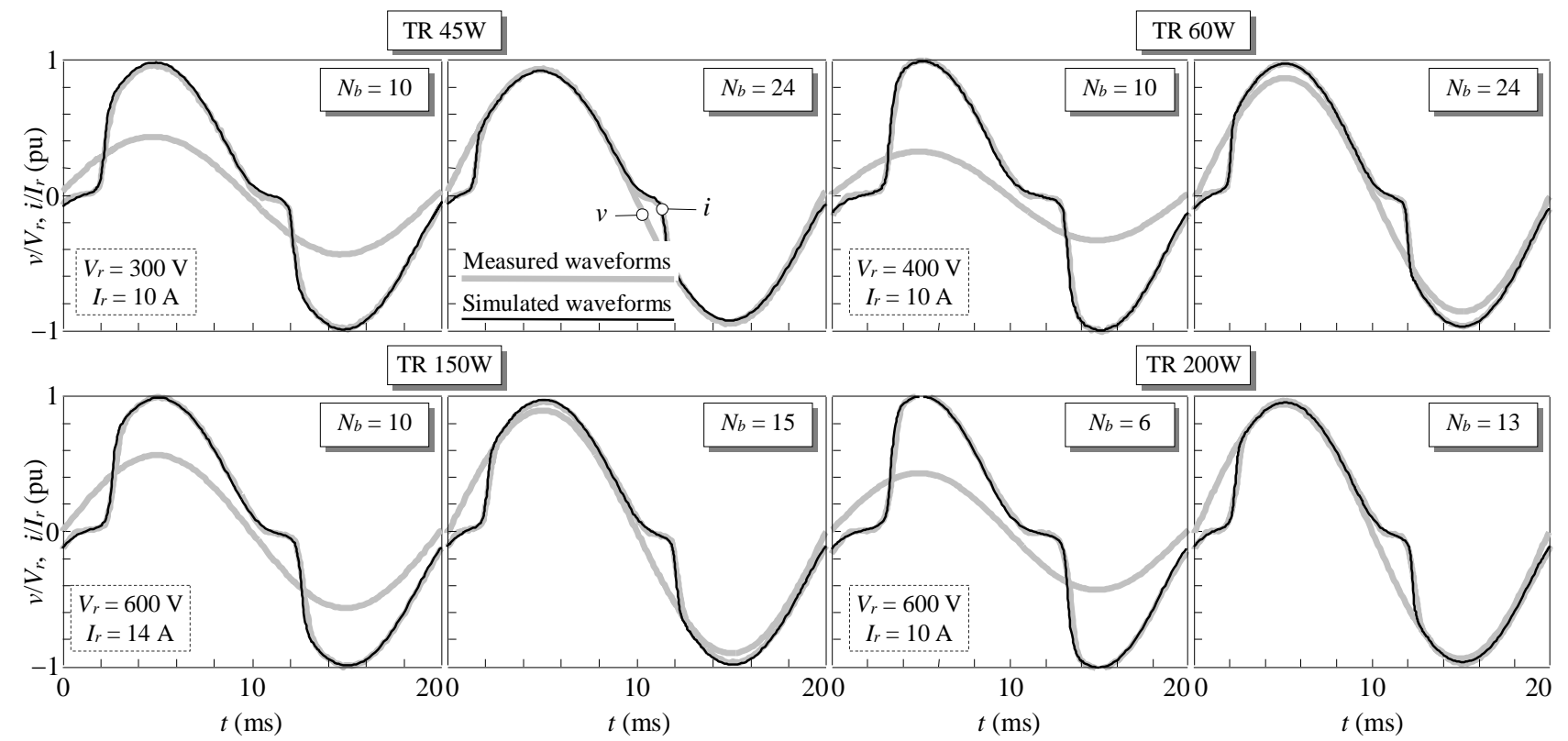

Fig. 11 Measured and simulated voltage and current waveforms of Test \# 2.

measured. Fig. 11 shows the measurements and simulations of this current. The circuit supply voltage was also measured and is plotted in Fig. 11 as a reference. The good accuracy of the proposed model is worth noting. The simulations were extended to $R_{\mathrm{Eq}}\left(N_{b}\right)$ values corresponding to $N_{b}$ values from 5 to 1000 in order to analyze the impact of the failed luminaires on current harmonic distortion depending on the AGL system size (i.e., depending on the number of AGL system luminaires). This could not be experimentally tested due to limitations of the laboratory power sources. Fig. 12 plots the total harmonic distortion THD of the simulated consumed currents as a function of the number of AGL system luminaires $N_{b}$,

$$
T H D=\frac{\sqrt{\sum_{h \neq 1} I_{h}^{2}}}{I_{1}},
$$

where $I_{1}$ and $I_{h}$ are the rms values of the fundamental and $h^{\text {th }}$ harmonic currents, respectively. These simulations reveal that smaller AGL systems have a larger impact on current harmonic distortion. The polynomials in Fig. 12 fit the THD curves, providing analytical functions to determine the current harmonic distortion from the AGL size in the event of luminaire burnt outs. The previous results could be very useful in AGL system studies and will be fully explored with field measurements in further research works. The THD of the experimental test current measurements in Fig. 11 is also plotted with dots in Fig. 12 to validate the transformer model results.

\section{Conclusions}

This paper presents an AGL transformer model, which considers transformer core saturation. The study is supported by extensive measurements on AGL transformers of different power ratings and trade names. The saturation phenomenon must be taken into account because AGL transformers operate in open circuit at rated current in the event of luminaire burnt out. An estimation procedure to obtain the transformer model parameters 

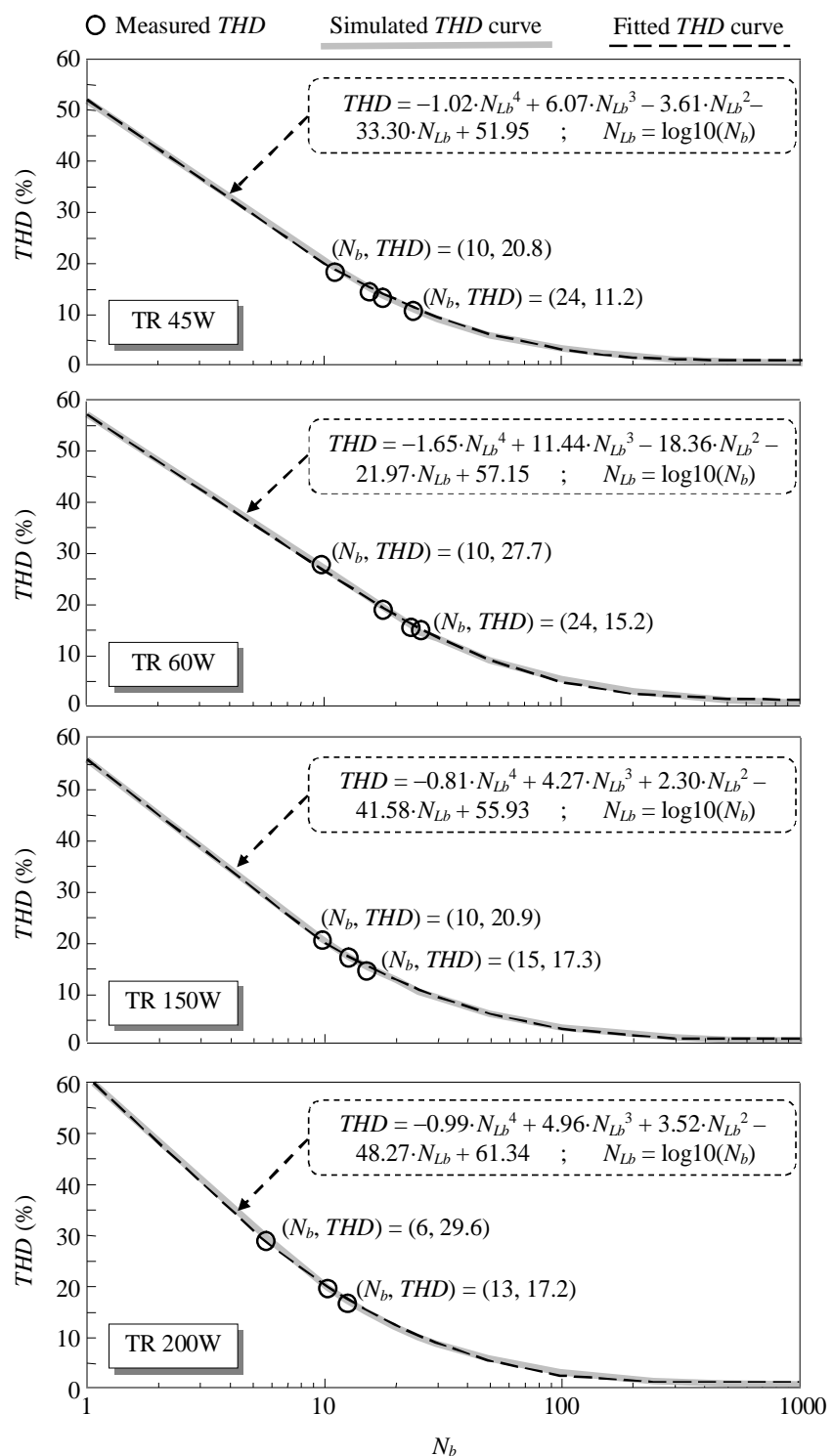

Fig. 12 Influence of failed luminaires on current harmonic distortion in AGL systems.

is also described. Both the AGL transformer model and the estimation procedure are necessary to analyze the behavior of AGL systems. The harmonic current pollution caused by failed luminaires is pointed out and its impact on harmonic distortion is analyzed. It is concluded that luminaire burnt out can lead to total harmonic distortions of the consumed current above $20 \%$ in small AGL systems. This can affect the correct operation of these systems as confirmed from different measurements on the AGL system of the airport of Reus (Spain). Therefore, it is necessary to have adequate models of the circuit elements that allow the simulation of AGL systems and the analysis of their behavior in order to prevent potential problems.

\section{Acknowledgements}

This research was carried out with the support of the A.A.A.S.A airport of Reus (Spain), which the authors gratefully acknowledge. 


\section{References}

1 Aerodrome design manual, Part 4: Visual aids, 4th Edition International Civil Aviation Organization, 2004.

2 Aerodromes, Volume I: Aerodrome design and operations, Annex 14 to the convention on International Civili Aviation, 4th Edition International Civil Aviation Organization, 2004.

3 Saraf, N., Salvi, R., Salunkhe, N., and Sahasrabuddh, R.: 'Airfield lamp monitoring \& control systems,' Int. Conf. on Information, Communication and Embedded Systems (ICICES 2013), February. 2013, pp. 1141-1143.

4 Kevin, P.E.: 'Integration of aviation lighting system and computer controlled monitoring system,' IEEE Int. Conf. on Systems, Man and Cybernetics, October 1996, pp. 1132-1137.

5 Granado, J., Chavez, J., Torralba A. and Oria, A. C.: 'Modeling airfield lighting systems for narrowband power-line communications,' IEEE Trans. on Power Delivery, 2010, 25, (4), pp. 2399-2405.

6 Smith, C. C.:'Some aspects of airport lighting,' Electronics and Power, 1970, 16, (3), pp. 85-89.

7 Zhongming, Y., Lo, E., Pong, M.H., Tang Ping, 'Power quality investigation of cluster of phase controlled rectifiers of airport grounding lighting system,' The 25th Annual Conference of the IEEE (IECON '99), December 1999, pp. 247252.

8 Bejleri, M., Rakov, V. A., Uman, M. A., Rambo, K. J., Mata C. T., and Fernandez, M. I.: 'Triggered lighting testing of an airport runway lighting system,' IEEE Trans. on Electromagnetic Compatibility, 2004, 46, (1), pp. 96-101.

9 Theethayi, N., Rakov, V. A., and Thottappillil, R.: 'Responses of airport runway lighting system to direct lighting strikes: Comparisons of TLM predictions with experimental data,' IEEE Trans. on Electromagnetic Compatibility, 2008, 50, (3), pp. 660-668.

10 Matlab 7.9 (R2009b) and Simulink, The MathWorks, Natick, MA: 2009

11 Fed. Aviat. Admin: Specification for Series to Series Isolation Transformers for Airport Lighting System (150/534547B), Airport Safety and Standards, 2005. 